\title{
Textbook of Oral and Maxillofacial Surgery
}

Author: Rajiv M Borle

Publisher: Jaypee Brothers Medical Publishers (P) Ltd

Language: English

ISSN: 9789351520092

Edition: 1/e

Publish Year: 2014

Pages: 830, illustrated

Price: $£ 78.00$

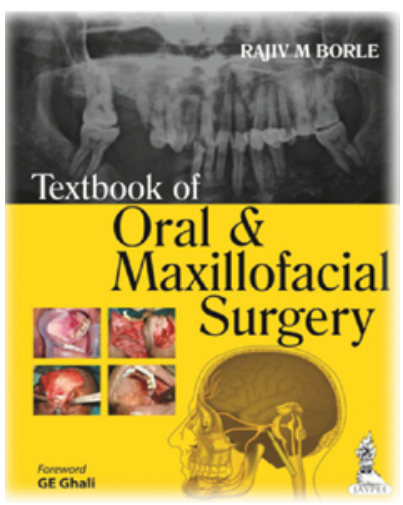

The Textbook of Oral and Maxillofacial Surgery by Professor Rajiv M Borle is a contemporary guide and a compilation of the authors' personal experiences. The book is divided into twelve sections and tackles the basic principles of surgery, neurological disorders of the face and general anesthesia, dentoalveolar surgeries, diseases of the maxillary sinus and salivary glands, infection and trauma of the maxillofacial region, facial deformities and TMJ disorders, neoplastic condition of the head, neck and face. At the end of each chapter all the incorporations have been authenticated by giving the necessary bibliography.

This first edition of the book addresses undergraduate and postgraduate students in the field of Oral and Maxillofacial Surgery, Otolaryngology, and Plastic Surgery. Presented in an easy-to-follow format, the text is enriched with clinical photographs, radiographic images, charts and a lot of illustrations to provide better understanding.

\footnotetext{
Florin-Eugen Constantinescu

DDS, PhD Student Romania e-mail:
} Holistic Dental \& Medical Institute of Bucharest-ROPOSTURO, Bucharest, dr.florin.constantinescu@gmail.com 